\title{
Dynamic Spray and Wait Routing Protocol for Delay Tolerant Networks
}

\author{
Longbo Zhang, Chen Yu, and Hai Jin \\ Services Computing Technology and System Lab \\ Cluster and Grid Computing Lab \\ School of Computer Science and Technology \\ Huazhong University of Science and Technology, Wuhan, 430074, China \\ \{yuchen, hjin\}@hust.edu.cn
}

\begin{abstract}
Delay Tolerant Networks (DTN) is one of the mobile wireless networks that cannot set up the end-to-end communication path between the source and destination nodes pair in most of time. In this paper, we propose an improved routing protocol named as Dynamic Spray \& Wait (DS\&W), which is based on Spray and Wait routing strategy attempting to gain the higher delivery ratio benefits of replication-based routing as well as the lower resource utilization benefits of forwarding-based routing. But different from S\&W, DS\&W routing strategy focus on dynamic control to reduce the scale of the messages' flooding by calculating the delivery success probabilities to destinations. DS\&W routing protocol can dramatically reduce the overhead ratio in DTNs. Simulation results also evident that DS\&W protocol outperforms other existing DTN routing protocols.
\end{abstract}

Keywords: Delay Tolerant Network (DTN), mobile networks, epidemic routing, Spray \& Wait routing, dynamic control.

\section{Introduction}

Delay Tolerant Networks (DTN) [1] often refer to sparse mobile ad hoc network, where a routing path does not necessarily exist. In DTNs, both nodes and links may be unreliable. Many emerging communication networks fall into this paradigm including wildlife tracking and habitat monitoring sensor networks (IPN) [2], interplanetary networks [3], nomadic communities networks [4], etc.

Many challenges affect the routing in DTNs such as the changing network topology, low delivery ratio and high delay. The problems can be mitigated by using flooding-based scheme or by predicting the network topology [5] to ensure the delivery ratio and delay. Many works shows that flooding-based scheme has a better performance on delivery ratio and delay than predicting scheme, but it will generate numbers of redundant copies and increase the overhead ratio which would have negative impact on the efficiency, if the flooding scale can not be controlled in a suitable scale, it would lead to network congestion. Thus, the efficiency of a flooding-based scheme DTN routing protocol relies essentially on the appropriate scale of message flooding. 
In this paper, we propose DS\&W, a routing protocol for DTNs, which dynamically control the scale of message flooding by predicting the delivery probabilities. The contributions presented in this paper are: we propose a suitable method to control the scale of message flooding; based on the dynamic control, we propose DS\&W, a novel routing protocol which relies on the control of the scale of flooding. With DS\&W protocol, the scale of message flooding is dynamically changeable, and different from other flooding-based scheme routing protocols, it could reduce the overhead ratio with little effect on delivery ratio and delay.

The paper is organized as follows. Section 2 presents the state of the art for DTN routing and the traditional method of the message flooding. Section 3 presents the dynamic control and DS\&W protocol. Section 4 provides the simulation results of DS\&W routing and related discussion. Finally, section 5 concludes the paper.

\section{Related Works}

Several routing protocols have been proposed for DTNs. These protocols can be classified into two categories: single-copy routing protocol [6] and multi-copy routing protocol [7]. With the single-copy routing protocols, only one copy of the message is transported by the topology knowledge of the network. In order to increase the delivery success probabilities, the topology knowledge should be predicted by historical encounters between nodes, location information or others [8]. The Direct Delivery protocol and the First Contact protocol are the use of this strategy. With the multi copy routing, the node carrying the message sends a copy to each encountered node. This is repeated until the destination receives the message. In this case, the contacts are assumed to be totally opportunistic. PRoPHET protocol [9], Epidemic Routing protocol [10], and Spray \& Wait routing protocol [11] are the use of this strategy. Due to the low delivery ratio and long delay of the single-copy routing, the multi-copy routing is the mainstream routing protocol.

PRoPHET protocol determines the delivery probability in each node, which is related to the history of the encounters. Transfer is done only for an encountered node, which has a good probability to deliver the message to its final destination. The delivery probability used by each node is recalculated according to three rules:

(a) When the node $\boldsymbol{M}$ encounters another node $\boldsymbol{E}$, the probability for $\boldsymbol{E}$ is increased:

$$
P(M, E)_{\text {new }}=P(M, E)_{\text {old }}+\left(1-P(M, E)_{\text {old }}\right) \times L_{\text {encounter }}
$$

where $L_{\text {encounter }}$ is an initialized constant.

(b) The predictabilities for all destinations $\boldsymbol{D}$ other than $\boldsymbol{E}$ are aged:

$$
P(M, D)_{\text {new }}=P(M, D)_{\text {old }} \times \gamma^{k}
$$

where $\gamma$ is the aging constant and $k$ is the number of time units that has elapsed since the last aging. 
(c) Predictabilities are exchanged between $\boldsymbol{M}$ and $\boldsymbol{E}$ and the transitive property of predictability is used to update the predictability of destinations $\boldsymbol{D}$ for which $\boldsymbol{E}$ has a value on the assumption that $\boldsymbol{M}$ is likely to meet $\boldsymbol{E}$ again:

$$
\begin{aligned}
P(M, D)_{\text {new }} & =P(M, D)_{\text {old }} \\
& +\left(1-P(M, D)_{\text {old }}\right) \times P(M, E) \times P(E, D) \times \beta
\end{aligned}
$$

where $\beta$ is a scaling constant.

In PRoPHET protocol, if a node $\boldsymbol{i}$ encounter another node $\boldsymbol{j}$, node $\boldsymbol{i}$ will decide whether to send the message to node $\boldsymbol{j}$ based on the delivery probability of them.

Epidemic routing can be described as following: the source node, which has $N$ neighbor nodes, sends $N$ copies of the message to its $N$ neighbor nodes. Afterwards, each neighbor node judges whether the received message copy has been received before or not. If it has, the neighbor node discards the copy; otherwise the neighbor node will keep this message copy and send the same copy to all its neighbor nodes until the message reaches the destination node. In the ideal situation, epidemic routing is considered to be the most effective strategy, but in reality, the overhead ratio is high and it is faced with serious network congestion.

In order to solve these problems, fixing the number of copies is the most common method. Spray \& Wait routing protocol uses this solution. In Spray \& Wait routing, there are two phases: spray phase and wait phase. In spray phase, the source node of a message initially starts with $\boldsymbol{L}$ copies of this message, which is related with the number of nodes and the requirements for delay. Any node $A$ that has $n>1$ message copies (source or relayed), when it encounters another node $B$ with no copies, it hands over to $B^{\lfloor n / 2\rfloor}$ copies and keeps $B^{\lceil n / 2\rceil}$ copies for itself; when $A$ is left with only one copy, it switches to direct transmission. $\boldsymbol{L}$ can be calculated from:

$$
\left(H_{M}^{3}-1.2\right) L^{3}+\left(H_{M}^{2}-\frac{\pi^{2}}{6}\right) L^{2}+\left(a+\frac{2 M-1}{M(M-1)}\right) L=\frac{M}{M-1}
$$

where $M$ is the number of nodes; $a$ is multiples of the time units; $H_{n}^{\tau}=\sum_{i=1}^{n} \frac{1}{i^{\tau}}$ is the $n^{\text {th }}$ Harmonic number of order of $\tau$.

The multi-copy routing protocol can achieve higher delivery ratio and lower delay than the single-copy routing protocols, but it will increase the overhead ratio and wastes energy. Although Spray \& Wait routing to a certain extent solves the above problems, these problems still exist. Considered Spray \& Wait routing, the number of copies $L$ is not appropriate in all cases such as the following situation: in spray phase, a message sends copies to other nodes after a period of time, set the number of nodes $L_{1}$, then it will continue to flooding message to the left node until the number of nodes reaches $L$; then the routing protocol goes in the wait phase. If the message is successfully forwarded by a node in $L_{1}$, we can consider the spray phase after $L_{1}$ is useless. 


\section{Dynamic Control and DS\&W Routing Protocol}

We can find the limitation of Spray \& Wait routing is that it has to fix the copies of message to control the overhead ratio; the spray phase will not stop until the message get to the destination or wait phase. The flooding scale $L$ related with the network size can guarantee the maximum delivery delay and stationary message flooding, but it will increase some messages' flooding. For some messages, a smaller $L$ can not only guarantee the delivery ratio and delay, but also decrease the overhead ratio. Since the redundant message flooding in spray phase, the routing efficiency drops. So, if the spray phase can be controlled dynamically, it will have a better performance than Spray \& Wait protocol. In order to ensure the delivery ratio and delay, the scale of message flooding should also not be too small. So how to ensure the changeable flooding scale appropriate is very important. Next, we will give our method using the probability of nodes to realize the dynamic control.

Considering PRoPHET routing protocol, it gives a method to evaluate the probability to deliver the message. In the spray phase, we can calculate the total probability of the nodes. When the probability is high enough to ensure the delivery ratio and delay while the spray phase is not end, we can force it end, so that the spray phase can be controlled dynamically. The following section will prove that our assumption is correct, the method of dynamic flooding is effectively. Based on the dynamic flooding, we will give the pseudo code of DS\&W routing protocol.

In order to prove our assumption, we do the following works. For the delay, we divided it into two parts: $T_{\mathrm{s}}$ and $T_{\mathrm{w}}$, means the spray phase and wait phase. It is certain that if we reduce the number of copies $T_{\mathrm{s}}$ will decrease and $T_{\mathrm{w}}$ will increase. Assume that the node can meet another node after $t_{\text {unit }}$, and the node's average delivery probability as $\bar{p}$. In spray phase, expect of $T_{\mathrm{s}}$ is: $T_{s}=\log _{2}^{L} t_{\text {unit }}$. In wait phase, all nodes carrying packets is connected with the destination with the probability of $1-(1-\bar{p})^{L}$, so expect of $T_{\mathrm{w}}$ is: $T_{w}=\frac{t_{\text {unit }}}{1-(1-\bar{p})^{L}}$. The delivery delay is:

$$
T=\log _{2}^{L} t_{\text {unit }}+\frac{t_{\text {unit }}}{1-(1-\bar{p})^{L}}
$$

If we decrease the number of nodes to $\lambda L(\lambda \in(0,1))$, then in spray phase, expect of $T_{\mathrm{s}}$ ' is: $T_{s}^{\prime}=\log _{2}^{\lambda L} t_{\text {unit }}$. In wait phase, when the number of node is $L$ if the transmission node is in the node of $\lambda L$, the wait phase is $T_{w}^{\prime}=\frac{t_{\text {unit }}}{1-(1-\bar{p})^{L}}$, if the node is not in the nodes of $\lambda L$, the message will be transported by the $\lambda L$ nodes, then $T_{w}^{\prime}=\frac{t_{\text {unit }}}{1-(1-\bar{p})^{\lambda L}}$, so the expect of $T_{\mathrm{w}}$ is: $T_{w}^{\prime}=\frac{\lambda t_{\text {unit }}}{1-(1-\bar{p})^{L}}+\frac{(1-\lambda) t_{\text {unit }}}{1-(1-\bar{p})^{\lambda L}}$. The delivery delay is: 


$$
\begin{gathered}
T^{\prime}=\log _{2}^{\lambda L} t_{\text {unit }}+\frac{\lambda t_{\text {unit }}}{1-(1-\bar{p})^{L}}+\frac{(1-\lambda) t_{\text {unit }}}{1-(1-\bar{p})^{\lambda L}} \\
\Delta T=T-T^{\prime}=\left[\log _{2}^{\frac{1}{\lambda}}+\frac{1-\lambda}{1-(1-\bar{p})^{L}}-\frac{1-\lambda}{1-(1-\bar{p})^{\lambda L}}\right] t_{\text {unit }}
\end{gathered}
$$

Derivative of $\Delta T$, we find that $\Delta T_{\lambda=1}^{\prime}<0$ and $\Delta T_{\lambda=0}^{\prime}>0$, the function graph is similar to a parabola. $\Delta T_{\lambda=1}^{\prime}=0$, which means there is a $\lambda$ that makes $\Delta T_{\lambda}>0$, and $T$ is smaller than $T$, and limit of $\Delta T_{\lambda=0}$ is negative infinity. So when $\lambda$ is close to 1 , the deliver delay is shorter than spray $\&$ wait routing, and there should be a minimal value of the delay. After that when $\lambda$ gets smaller, the delay gets larger. By calculating the total probability, we can make the $\lambda$ in a suitable scope. By the research, we reach the following conclusions: with the dynamic control, the routing reduces the overhead while has little influence on the delivery ratio and delay.

By the calculation and analysis, the routing forwarding algorithm can be described as: (1) maintain the delivery probability of each node which is related to the history of the encounters; (2) send messages to its neighbor node, calculate the total delivery probability when nodes are connection, and update the record of the messages and the number of copies; (3) if the total delivery probability is higher than $p_{\max }$ or $L$ is equal to 1 , switches to wait phase. Next is the pseudo code for DS\&W forwarding algorithm:

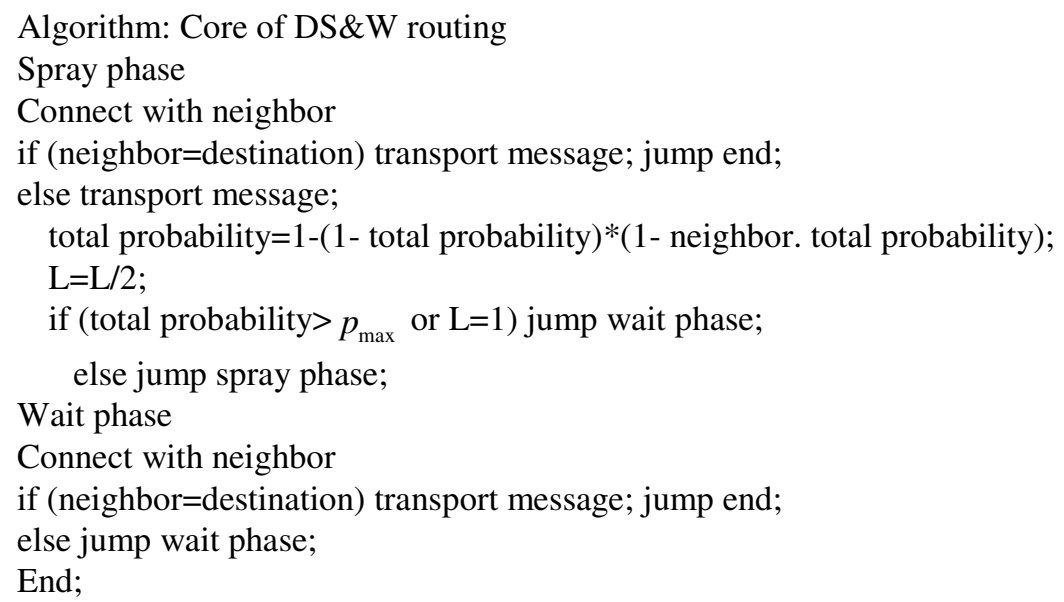

\section{Performance Evaluation}

To demonstrate and evaluate the performance of DS\&W, we use ONE1.4.1 [12], an Opportunistic Network Environment simulator which provides a powerful tool for 
generating mobility traces, running DTN messaging simulations with different routing protocols, and visualizing both simulations interactively in real-time and results after their completion. 125 nodes are distributed in Helsinki, capital of Finland, about $448 \mathrm{~km}^{2}$, the nodes are divided into four groups. Group 1 and 4 simulate the pedestrians' nodes, they all have $10 \mathrm{MB}$ memory, $1 \mathrm{Km} / \mathrm{h}$ move speed, $100 \mathrm{~m}$ transmit range, but move in different area. Group 2 and 3 simulate the car nodes, they have $15 \mathrm{MB}$ memories, $150 \mathrm{~m}$ transmit range, move only on the rode of city, but have different speeds: $2 \mathrm{Km} / \mathrm{h}$ and $3 \mathrm{Km} / \mathrm{h}$. The flowing figures show the performance comparison of three routing protocols.

Table 1. Delivery-ratio of DS\&W routing and $S \& W$ routing

\begin{tabular}{|l|l|l|l|l|l|l|l|}
\hline Routing & $\begin{array}{l}\text { DS\&W- } \\
0.98\end{array}$ & $\begin{array}{l}\text { DS\&W- } \\
0.95\end{array}$ & $\begin{array}{l}\text { DS\&W- } \\
0.93\end{array}$ & $\begin{array}{l}\text { DS\&W- } \\
0.9\end{array}$ & $\begin{array}{l}\text { DS\&W- } \\
0.85\end{array}$ & $\begin{array}{l}\text { DS\&W- } \\
0.8\end{array}$ & S\&W \\
\hline $\begin{array}{l}\text { Delivery- } \\
\text { ratio }\end{array}$ & 0.4508 & 0.4486 & 0.4545 & 0.4494 & 0.4228 & 0.4169 & 0.4649 \\
\hline
\end{tabular}

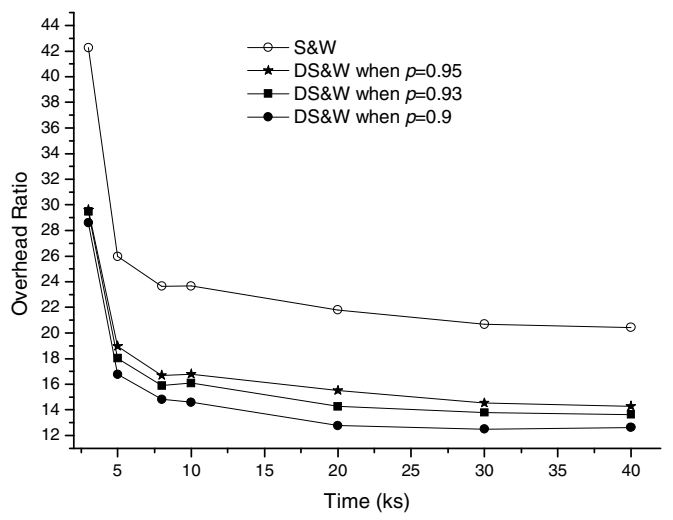

Fig. 1. Overhead ratio comparison between DS\&W and S\&W

Table 1 shows the delivery ratio of S\&W routing and DS\&W routing with different $p_{\text {max }}$. We find that when the total probability is from 0.9 to 1 , the delivery ratio is similar to S\&W routing, this corresponds with our conclusion: if the total probability is high enough, there is negligible affection on delivery ratio. If the total probability gets lower, the delivery ratio would have slightly bad performance.

Figure 1 and 2 show the performance comparison between DS\&W routing and $\mathrm{S} \& \mathrm{~W}$ routing. We can find that when $p=0.95$, the overhead ratio reduces about $30.07 \%$, and the delay reduces about $5 \%$; when $p=0.93$, the overhead ratio reduces about $33.24 \%$, and the delay is basically unchanged; when $p=0.9$, the overhead ratio reduces about $38.22 \%$, and the delay increases about $13 \%$. It shows a good performance in reducing the burden of network and the delay corresponds with our conclusion: when $\lambda$ is close to 1 , the deliver delay is shorter than Spray \& Wait routing, and there should be a minimal value of the delay, after that when $\lambda$ gets smaller, the delay 
gets larger. So, when $p_{\max }$ is higher than 0.93 , the routing can reduce overhead ratio obviously without influencing the delay, when it is from 0.93-0.9, it will increase the delay, but it is still acceptable.

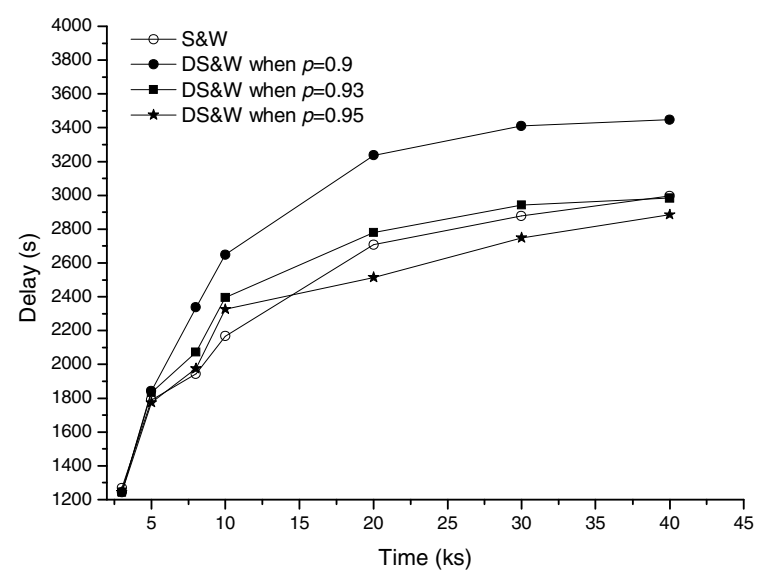

Fig. 2. Delay comparison between DS\&W and S\&W

Further, we consider the situation when $p_{\max }=0.85$, overhead ratio reduces about $49.5 \%$, and the delay increases about $42.4 \%$. If the network is troubled by the network congestion and is not stringent on delay, this performance still can be acceptable; but when $p$ get lower to 0.8 , the overhead ratio reduces about $58.75 \%$, the delay increases about $61.04 \%$, this is unacceptable. So, if $p$ gets smaller, the routing should be regarded as failed.

Table 2. Comparison of low p-DS\&W and S\&W

\begin{tabular}{|c|c|c|c|}
\hline Routing & DS\&W-0.85 & DS\&W-0.8 & S\&W \\
\hline Overhead-ratio & 10.3094 & 8.4273 & 20.4340 \\
\hline Delay (ms) & 4267.6299 & 4809.0593 & 2996.4775 \\
\hline
\end{tabular}

Through our analysis, we can summarize that when $p \in(0.9,1)$, the DS\&W routing shows a good performance in reducing the burden of network while with little influence to delay; when $p \in(0.85,0.9)$, the DS\&W routing protocol can be suitable for the network that is troubled by the burden of network and not particularly stringent requirements on delay.

\section{Conclusions}

In this paper, we propose a new routing protocol, named DS\&W, which is suitable for mobile delay tolerant networks. DS\&W routing protocol is based on multi-copy routing protocol and predicts probabilistic using history of encounters and transitivity. DS\&W uses the total probability of the nodes carrying message to dynamically 
control the number of copies. In this way, the overhead-ratio can be reduced, and delivery-ratio and delay will not change much. Simulation results show that DS\&W routing protocol outperforms Spray \& Wait routing protocol.

Acknowledgement. The work is supported by National Natural Science Foundation of China (No.61003220), Research Fund for the Doctoral Program of Higher Education of China (No.20090142120025), Fundamental Research Funds for the Central Universities (HUST:2010QN051) and Natural Science Foundation of Hubei Province of China (No.2010CDB02302).

\section{References}

1. Delay tolerant networking research group, http://www. dtnrg.org

2. Juang, P., Oki, H., Wang, Y., Martonosi, M., Peh, L.S., Rubenstein, D.: Energy-efficient Computing for Wildlife Tracking: Design Tradeoffs and Early Experiences with Zebranet. In: Proceedings of 10th International Conference on Architectural Support for Programming Languages and Operating Systems, pp. 96-107. ACM Press, California (2002)

3. Burleigh, S., Hooke, A., Torgerson, L., Fall, K., Cerf, V., Durst, B., Scott, K.: Delaytolerant Networking: An Approach to Interplanetary Internet. IEEE Communications Magazine 41, 128-136 (2003)

4. Doria, A., Udn, M., Pandey, D.P.: Providing Connectivity to the Saami Nomadic Community. In: Proceedings of 2nd International Conference on Open Collaborative Design for Sustainable Innovation (2002)

5. Singh, J.P., Dutta, P.: Temporal Behavior Analysis of Mobile ad hoc Network with Different Mobility Patterns. In: Proceedings of International Conference on Advances in Computing, Communication and Control, pp. 696-702. ACM Press, Mumbai (2009)

6. Spyropoulos, T., Psounis, K., Raghavendra, C.S.: Single-copy Routing in Intermittently Connected Mobile Networks. In: Proceedings of 1st IEEE Communications Society Conference on Sensor and Ad Hoc Communications and Networks, pp. 235-244. IEEE Press, Santa Clara (2004)

7. Spyropoulos, T., Psounis, K., Raghavendra, C.S.: Multiple-copy Routing in Intermittently Connected Mobile Networks. Technical Report, USC (2004)

8. Chen, X., Murphy, A.L.: Enabling Disconnected Transitive Communication in Mobile ad hoc Networks. In: Proceedings of Workshop on Principles of Mobile Computing collocated with PODC 2001, ACM Press, Rhode Island (2001)

9. Lindgren, A., Doria, A., Schelen, O.: Probabilistic Routing in Intermittently Connected Networks. SIGMOBILE Mobile Computing and Communications Review 7(3), 19-20 (2003)

10. Vahdat, A., Becker, D.: Epidemic Routing for Partially Connected ad hoc Networks. Technical Report CS-200006, Duke University (2000)

11. Spyropoulos, T., Psounis, K., Raghavendra, C.S.: Spray and Wait: An Efficient Routing Scheme for Intermittently Connected Mobile Networks. In: Proceedings of 2005 ACM SIGCOMM Workshop on Delay-Tolerant Networking, pp. 252-259. ACM Press, New York (2005)

12. Opportunistic Network Environment (ONE), http://www. netlab.tkk. fi/tutkimus/dtn/theone/ 